\title{
Green exfoliation of graphite flakes to efficiently synthesize
}

\section{Biographene}

\section{Noelia Losada ${ }^{\mathrm{a}}$, Angel Berenguer-Murciab ${ }^{\mathrm{b}}$, Diego Cazorla-Amorós ${ }^{\mathrm{b}}$ and Jose M. Palomo*a}

${ }^{\text {a }}$ Department of Biocatalysis. Institute of Catalysis (CSIC). Marie Curie 2. Cantoblanco.

Campus UAM, 28049 Madrid, Spain. Fax: +34-91-585-4760.

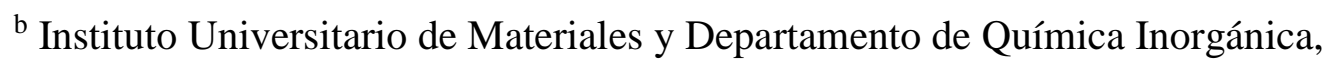
Universidad de Alicante, Apartado 99, San Vicente del Raspeig, E-03080 Alicante, Spain

E-mail: josempalomo@icp.csic.es 


\begin{abstract}
A new simple, rapid, and efficient methodology to produce undamaged graphene sheets from graphite flakes in water by a bio-exfoliation technology is described.

The methodology consists in the application of a lipase, with a very exclusive mechanism of interaction with hydrophobic surfaces, combined with a previous mechanical sonication, to selectively generate biographene in water. The adsorption of the lipase on the graphene sheets permits to keep the sheets separated in comparison with other methods. It is possible to obtain more than $80 \%$ of graphene (in the form of Few Layer Graphene) from low-cost graphite and with less damage compared to commercial graphene oxide (GO) or reduced GO. Experimental analysis demonstrated the formation of bilayer graphene mainly using lipase from Thermomyces lanuginosus (TLL).
\end{abstract}




\section{Introduction}

Graphene is a material with excellent electrical and thermal properties conjugated with very high mechanical strength and elasticity which make for a very broad applicability in areas such as electronics, materials, biomedicine, biotechnology, etc. ${ }^{1-13}$

Production of graphene has been performed by scotch tape peeling, ${ }^{14}$ different chemical and thermal strategies, ${ }^{15-18}$ and solvent/surfactant assisted exfoliation of graphite with sonication. ${ }^{19-20}$

In particular, the synthesis of graphene by the chemical reduction of graphene oxide (GO) by the Hummers method represents at present the most scalable method for the production of few layer graphene. ${ }^{15,17}$ However, this technique requires very harsh and toxic conditions, which probably causes defects within the graphene sheets, compromising properties and morphology. ${ }^{20}$

In this way, in recent years, strategies to synthesize graphene by a more sustainable way have been reported. The direct exfoliation of graphite to graphene in water seems to have the best outcome. ${ }^{21-28}$ By this route, a few methodologies have been described to produce biographene using different small molecules (aromatic compounds, carbohydrates), ${ }^{21-23}$ nanofibers, ${ }^{24}$ surfactants, ${ }^{25}$ or plants. ${ }^{26}$

In particular, the application of proteins for the exfoliation of graphite presents an interesting alternative. The application of some mechanical step to generate enough space between graphene layers in graphite is mandatory for the intercalation of proteins, considering that the interlayer distance between graphene layers $0.33 \mathrm{~nm}$ and the protein size being around 5-10 nm. A few examples have proven to be successful in the application of proteins. ${ }^{27-28}$ However, in these cases, proteins come from animal sera or are not pure. This is a disadvantage in order to obtain a completely selective covered 
surface. Heterogeneous samples are usually obtained using these protein mixtures, where a homogeneous surface coverage should be mandatory. Another important issue is the high efficiency exfoliation of graphite into single- or few-layered nanoplates, which remains a grand challenge and becomes the bottleneck in fundamental studies and applications of graphene.

At present the most specific methodology is represented by using hydrophobins, which present a hydrophobic part and another hydrophilic. ${ }^{29}$ It is demonstrated that they can interact by the hydrophobic area on graphene. Nevertheless, these proteins are very difficult to manipulate, biochemical production is complex producing very low yields and it has been shown that mixtures of graphene and graphite are obtained following this strategy. ${ }^{28}$

Therefore, the development of a highly selective methodology to produce a completely homogeneous modification of the graphene protein surface is mandatory. Also, from an economical point of view, the availability of the enzyme is critical.

This way, the use of lipases can solve these drawbacks. Lipases are enzymes with a particular structure based on their natural function - fats hydrolysis-. In nature, they work in the interface between oil and water, specifically adsorbed on the hydrophobic interface. ${ }^{30}$ This is possible because they present an oligopeptide lid which moves, generating a highly hydrophobic pocket, as an open conformation -minoritary- in homogeneous aqueous medium- ${ }^{31}$ which exists in equilibrium with the main conformation (closed conformation) where the lid moves covering the hydrophobic pocket generating a completely hydrophilic surface (Figure 1).

This equilibrium in water is completely shifted to the open conformation in the presence on a hydrophobic interface, solving the open conformation on the hydrophobic interface from the hydrophilic lipase in water. ${ }^{32-35}$ 


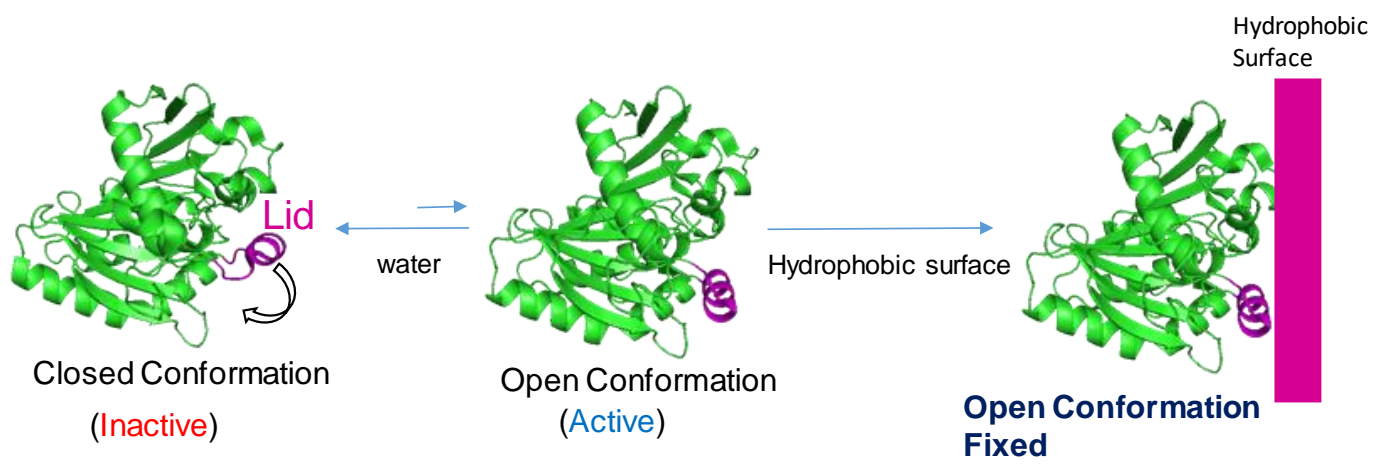

Figure 1. Mechanism and interaction of lipases with hydrophobic surfaces.

This capacity has been demonstrated to work in the presence of different hydrophobic interfaces (detergents, solid materials, hydrophobic proteins) ${ }^{32-35}$ (Figure 2).

Recently a lipase interacting with graphene has been published although in all cases as a covalent immobilization technique on graphene oxide using crosslinkers. ${ }^{36-37}$

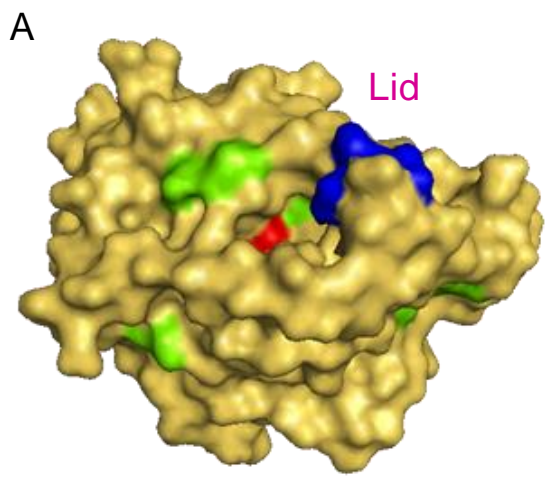

B

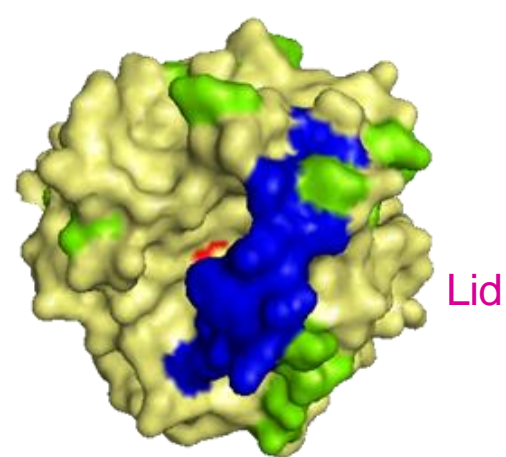

Figure 2. Hydrophobic area and Lid of A) CAL-B, B) TLL. Lid (blue)

In this work we applied for the first time the special selective capacity of these lipases for their selective adsorption on graphene from graphite flakes. These represent a very fast, cost-efficient and sustainable methodology of bioexfoliation of graphite to graphene. These enzymes can be homogeneously located on the graphene surface, permitting their 
direct production from the graphite present in a water suspension. This also represents a method for the specific functionalization of graphene, because we can selectively control the enzyme location, thus controlling the number of groups (amino, carboxyl) for the modification of graphene.

Furthermore, we demonstrate how the capacity of the lid has influence on the final results and how it is possible to obtain biographene very easily on a large scale. 


\section{Materials and Methods}

\subsection{Materials}

Graphite flakes and p-nitrophenilpropionate (pNPP) were purchased from Sigma-Aldrich Co (St Louis, MO, USA). Lipase B from Candida antarctica (CAL-B) solution and lipase from Thermomyces lanuginosus (TLL) were obtained from Novozymes (Copenhagen, Denmark). Aqueous solutions were prepared using UltraPure ${ }^{\mathrm{TM}}$ DNase/ RNase-Free Distilled Water (Thermo Fisher Scientific). Inductively coupled plasma - optical emission spectrometry (ICP-OES) was performed in an OPTIMA 2100 DV instrument (PerkinElmer, Waltham, MA, USA). X-Ray diffraction (XRD) patterns were obtained using a Texture Analysis D8 Advance Diffractometer (Bruker, Billerica, MA, USA) with $\mathrm{Cu} \mathrm{K} \alpha$ radiation. Transmission electron microscopy (TEM) and high resolution TEM microscopy (HRTEM) images were obtained on a 2100F microscope (JEOL, Tokyo, Japan). Interplanar spacing in the nanostructures was calculated by using the inversed Fourier transform with the GATAN digital micrograph program (Corporate Headquarters, Pleasanton, CA, USA). Scanning electron microscopy (SEM) imaging was performed on a TM-1000 microscope (Hitachi, Tokyo, Japan). Vibra-Cell ${ }^{\mathrm{TM}}$ Ultrasonic Liquid Processors VCX130 (Sonics) was used for graphite sonication process. Raman spectra were collected at room temperature using a dispersive JASCO NRS-5100 equipment with a focal distance of $300 \mathrm{~mm}$. The laser used in the experiments was a solidstate laser $(532 \mathrm{~nm})$ with a sample output power of $4 \mathrm{~mW}$. The equipment is coupled to a MPLFLN 20x UV objective. Prior to analysis the Raman shift was calibrated using the $520 \mathrm{~cm}^{-1}$ band of a pure silicon sample. Two 60 -second scans were performed for each spectrum. The obtained spectra were baseline corrected in order to remove any background fluorescence. To recover the biohybrids, a Biocen 22 R (Orto-Alresa, Ajalvir, 
Spain) refrigerated centrifuge was used. Spectrophotometric analyses were run on a V730 spectrophotometer (JASCO, Tokyo, Japan).

\subsection{Ultrasonication of graphite flakes.}

- Method 1: Simple Sonication of graphite flakes.

The graphite flakes $(1 \mathrm{~g})$ are added together with $20 \mathrm{~mL}$ of distilled water to a $50 \mathrm{~mL}$ centrifuge tube ( $50 \mathrm{~mL}$ Falcon tube). Then, the graphite is exfoliated by alternating cycles of 5 min sonication / rest, for 30 minutes at an amplitude of $80 \%$. To avoid excessive heating of the mixture, the $50 \mathrm{~mL}$ Falcon tube is introduced into a mixture of ice and cold acetone. This exfoliation allows the weakening of the bonds between sheets. The nonexfoliated graphite is separated from the mixture by centrifugation. The mixture was transferred to $15 \mathrm{~mL}$ centrifuge tubes ( $15 \mathrm{~mL}$ Falcon tubes) and centrifuged at $400 \mathrm{rpm}$ for $1 \mathrm{~min}$. The supernatant is carefully separated from the unfolded graphite solid. Finally, it was placed in a Petri dish and dried in the stove at $50^{\circ} \mathrm{C}$. The method allows obtaining $100 \mathrm{mg}$ of graphene, final yield of $10 \%$.

- Method 2: Double Sonication of graphite flakes.

The graphite flakes $(1 \mathrm{~g})$ are added together with $20 \mathrm{~mL}$ of distilled water to a $50 \mathrm{~mL}$ centrifuge tube (50 mL Falcon tube). Once this is done, the graphite is exfoliated by alternating cycles of 5 min sonication / rest, for 1 hour at an amplitude of $80 \%$. To avoid excessive heating of the mixture, the $50 \mathrm{~mL}$ Falcon tube is introduced into a mixture of ice and cold acetone. This exfoliation allows the weakening of the bonds between sheets. The non-exfoliated graphite is separated from the mixture by centrifugation. The mixture was transferred to $15 \mathrm{~mL}$ centrifuge tubes $(15 \mathrm{~mL}$ Falcon tubes) and centrifuged at 400 rpm for $1 \mathrm{~min}$. The black supernatant was carefully separated from the graphite solid. 
Finally, it was placed in a Petri dish and dried in the stove at $50^{\circ} \mathrm{C}$. The method allows obtaining $300 \mathrm{mg}$ of graphene, final yield of $30 \%$.

\subsection{Exfoliation of graphite flakes using lipase.}

- Method 3: Double sonication of graphite flakes + selective lipase adsorption

The graphite flakes $(1 \mathrm{~g})$ in $20 \mathrm{~mL}$ of water were sonicated as described in method 2 . After that, commercial lipase solution of TLL $(0.43 \mathrm{~mL})$ or commercial lipase solution of CALB (1 mL), in both cases offering $5 \mathrm{mg}$ lipase, were directly added to that and allowed to stir for a period of time between $30 \mathrm{~min}$ and $1 \mathrm{~h}$. The selective adsorption of the lipase was followed by measuring the supernatant using the pNPP activity assay (described below). After lipase immobilization, the suspension turned cloudy black. Then, the mixture was transferred to $15 \mathrm{ml}$ Falcon centrifuge tubes and centrifuged at $400 \mathrm{rpm}$ for 1 minute to remove some non-exfoliated graphite. After that, the $20 \mathrm{~mL}$ black suspension was centrifuged at $8000 \mathrm{rpm}$ for $10 \mathrm{~min}$ and then the water was removed. One $\mathrm{mL}$ of acetone was added to dissolve the black powder and then this mixture was transferred to a Petri dish and dried at $50^{\circ} \mathrm{C}$ for 4 hours. The method allowed obtaining $800 \mathrm{mg}$ of biographene-1, final yield of $80 \%$ using TLL and $600 \mathrm{mg}, 60 \%$ yield using CAL-B.

- Method 4: Double sonication of graphite flakes in the presence of lipase.

The graphite flakes $(1 \mathrm{~g})$ were added to a solution of $20 \mathrm{~mL}$ of water to which $0.43 \mathrm{~mL}$ of commercial lipase were previously added in the case of TLL or $1 \mathrm{~mL}$ in the case of CAL$\mathrm{B}$, (enzymatic loading: $5 \mathrm{mg}_{\text {enzyme }} / \mathrm{g}_{\text {support}}$ ). Then this mixture was sonicated using the aforementioned method 2. After that, the mixture was transferred to $15 \mathrm{~mL}$ Falcon centrifuge tubes and centrifuged at $400 \mathrm{rpm}$ for $1 \mathrm{~min}$. The supernatant was carefully separated from the unfolded graphite solid. After that, the $20 \mathrm{~mL}$ black suspension was 
centrifuged at $8000 \mathrm{rpm}$ for $10 \mathrm{~min}$ and then the water was removed. One $\mathrm{mL}$ of acetone was added to dissolve the black powder and then this mixture was transferred to a Petri dish and dried at $50^{\circ} \mathrm{C}$ for 4 hours. The method allowed obtaining $500 \mathrm{mg}$ of biographene-2, final yield of $50 \%$.

\subsection{Hydrolytic activity assay of pNPP}

This assay was performed by measuring the increase in the absorbance at $348 \mathrm{~nm}$ produced by the release of p-nitrophenol in the hydrolysis of $0.4 \mathrm{mM} \mathrm{pNPP}$ in $25 \mathrm{mM}$ sodium phosphate buffer at $\mathrm{pH} 7$ and $25{ }^{\circ} \mathrm{C}$. To initialize the reaction, $20 \mu \mathrm{l}$ of lipase solution or suspension were added to $2.5 \mathrm{ml}$ of substrate solution. One international unit of pNPP activity was defined as the amount of enzyme that is necessary to hydrolyze 1 $\mu \mathrm{mol}$ of pNPP per minute (IU) under the conditions described previously. 


\section{Results and discussion}

\section{Characterization of graphene flakes.}

To make an identification and comparison of the different methods used for the bioexfoliation of the graphite to obtain graphene, the characterization of the commercial flakes of graphite has been performed (Figure 3).

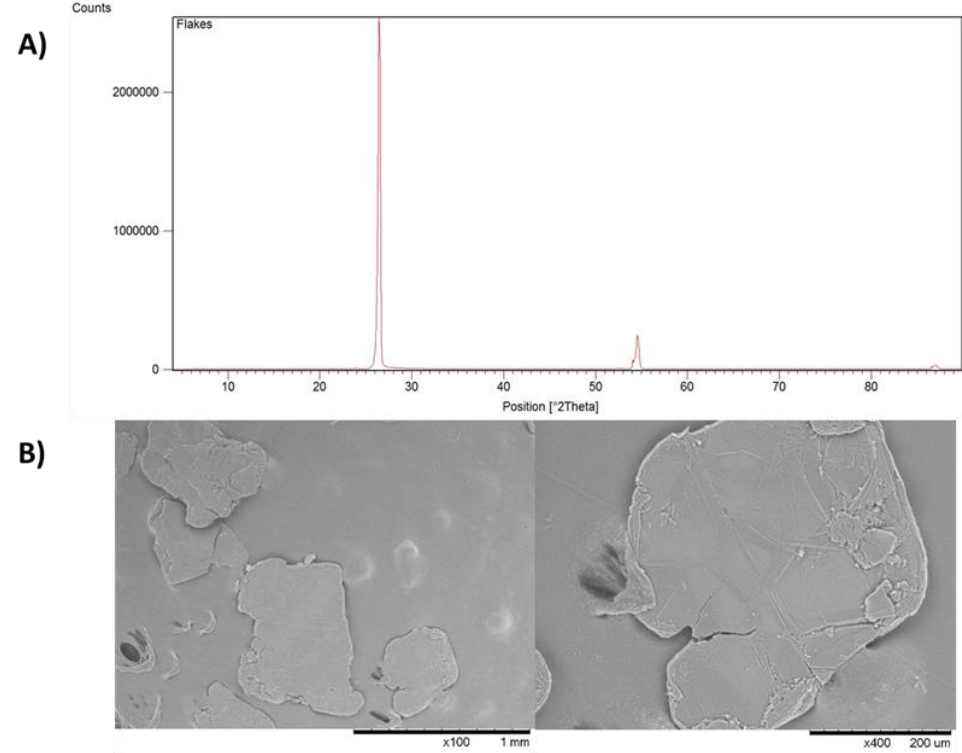

Figure 3. Characterization of graphite flakes. A) XDR; B) SEM images.

In Figure 3A, XRD pattern of the graphite is represented, where the peaks are identified as previously reported. ${ }^{38}$

Regarding the SEM images, the morphology and size of the graphite flakes, which presents approximately $1 \mathrm{~mm}$ (Figure 3B).

\section{Synthesis and Characterization of bio-graphene}

Four different methods have been performed in order to produce graphene from cheap graphite flakes. The simple mechanical sonication (method 1) and the double sonication 
(method 2) of graphite flakes in distilled water were developed as a first step in the process of obtaining bio-graphene based on the application of a mechanical force through ultrasound to the flakes of graphite. This strategy gave moderate to low yields of graphene (black suspension) of 10 to $30 \%$, respectively.

If we focus on the observed differences between method 1 (Simple Sonication) and method 2 (Double sonication), for example in the SEM images, that material presented a larger flake size by using method 1 (Figure 4A.1 and 4B.1) than by double mechanical approach (Figure 4A.2 and 4B.2). TEM analysis confirmed that the material obtained from method 1 showed a higher density solid without forming sheets (Figure 4C.1) whereas after application of method 2, a much less dense sample was observed and in which the number of layers can be appreciated (Figure 4C.2). These results seem to indicate the need for applying ultrasound for a longer time on the sample, so that progress was made on method 2. 

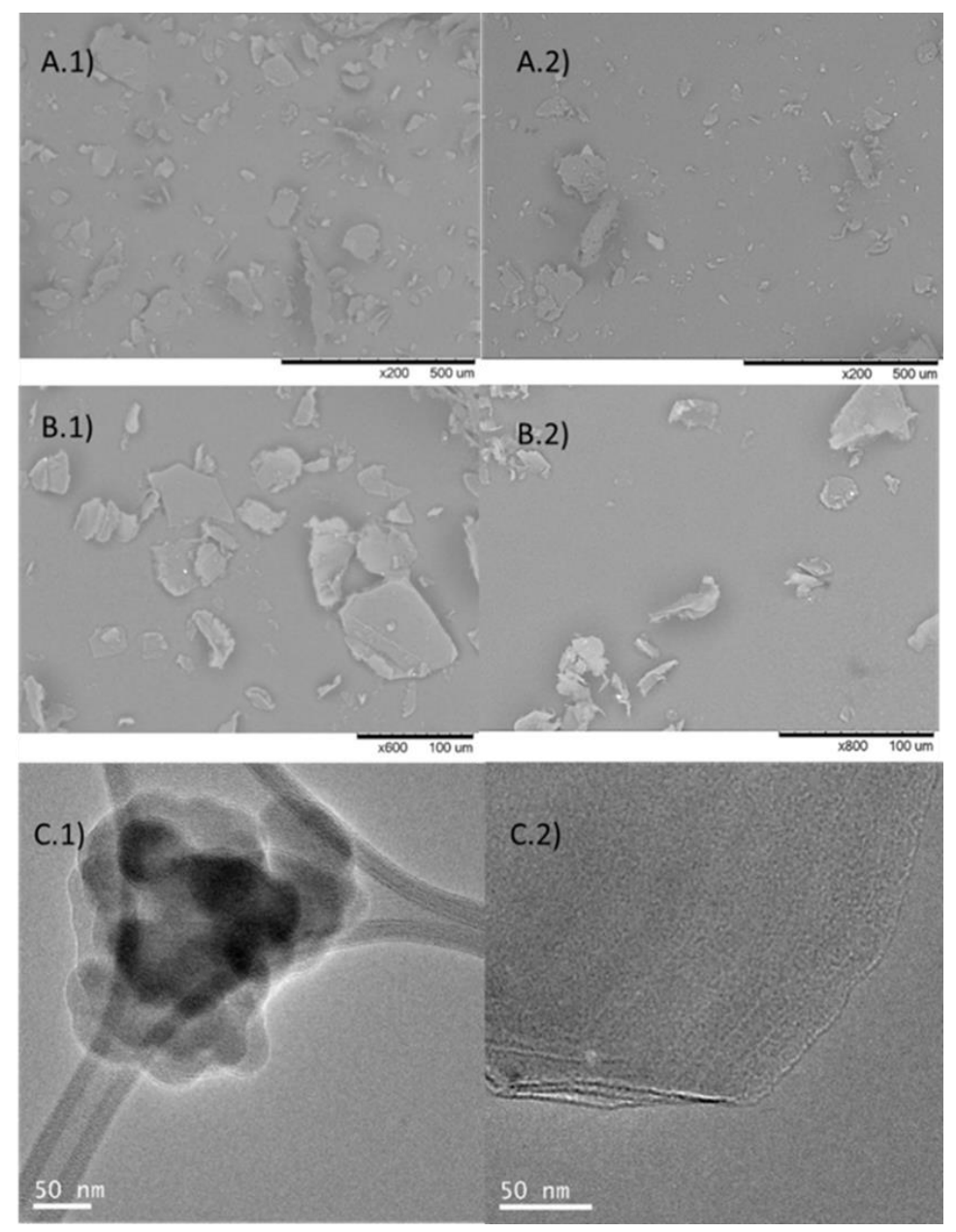

Figure 4. A) SEM images: A.1) Method 1, A.2) Method 2; B) SEM images: B.1) Method 1, B.2) Method 2; C) TEM images: C.1) Method 1, C.2) Method 2.

The method 3 consisted in combining the mechanical sonication of method 2 with the application of a biomolecule, a lipase (Figure 5). After a mechanical force was applied by ultrasound to graphite, the commercial liquid solution of lipase from $C$. antarctica $B$ (CAL-B) or T. lanuginosus (TLL) was directly added to the mixture. The lipase (loading of $5 \mathrm{mg}$ enzyme /g support) was fully adsorbed to the solid material at room temperature. The spacing obtained previously between the graphite sheets allows the lipase to be placed between them, giving rise to hydrophobic sheet-lipase interactions (interfacial adsorption) that permits separating said sheets obtaining graphene (Figure 5). 

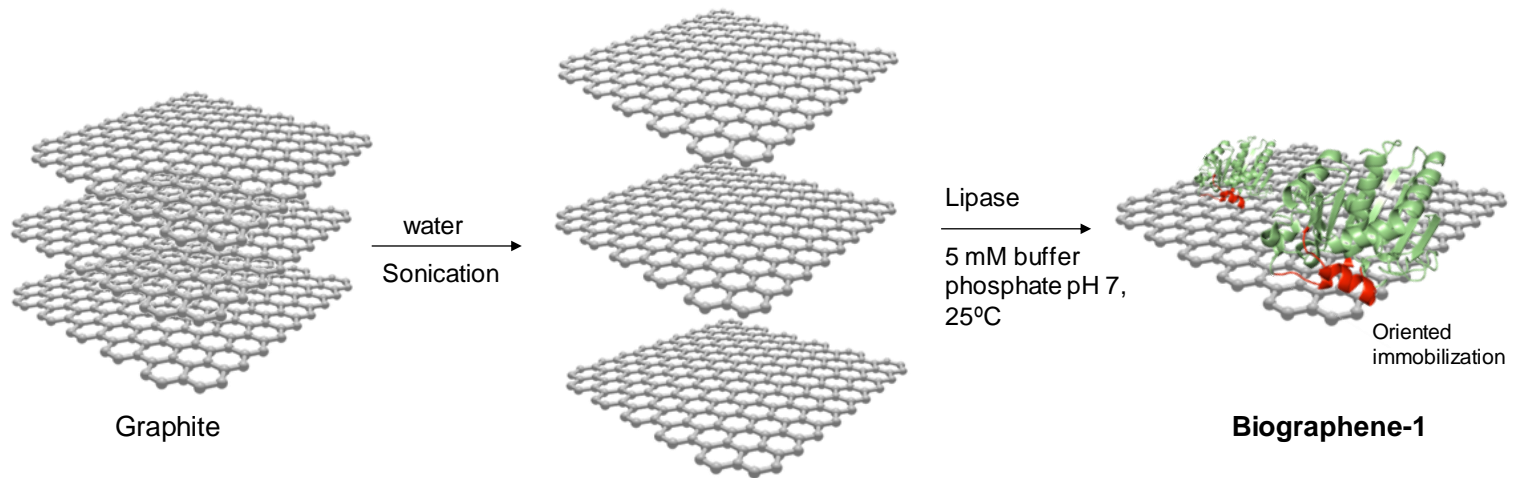

Biographene-1

Figure 5. Schematic representation of Biographene-1 preparation by method 3 .

The $100 \%$ immobilization was determined by measuring the enzymatic activity using the pNPP assay of the supernatant and was achieved after 30 min for TLL whereas with CALB $1 \mathrm{~h}$ of incubation was necessary. Also after recovery of the final biographene-1, the yield was higher using TLL than CAL-B, $80 \%$ against $60 \%$.

These phenomena could be explained considering the aminoacid composition and tridimensional structure of both lipases. Although both enzymes have similar molecular weight, TLL presents a much higher hydrophobic area than CAL-B (Figure 2), which has an influence on the immobilization rate of the open confirmation of the lipase on the graphene surface and also on the final interaction between enzyme and support. At first glance, the differences were evident, the aqueous suspension of graphene obtained using TLL was a homogeneous black solution while in CAL-B, although the suspension was black, a black solid slip towards the bottom was observed. These differences are also defined in SEM and TEM analysis (Figures 6-7). 
A

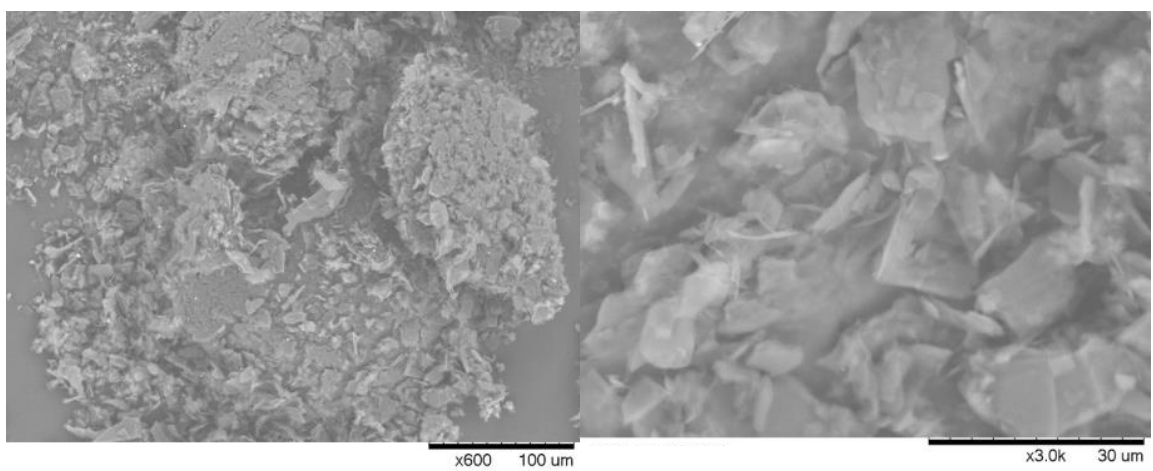

B
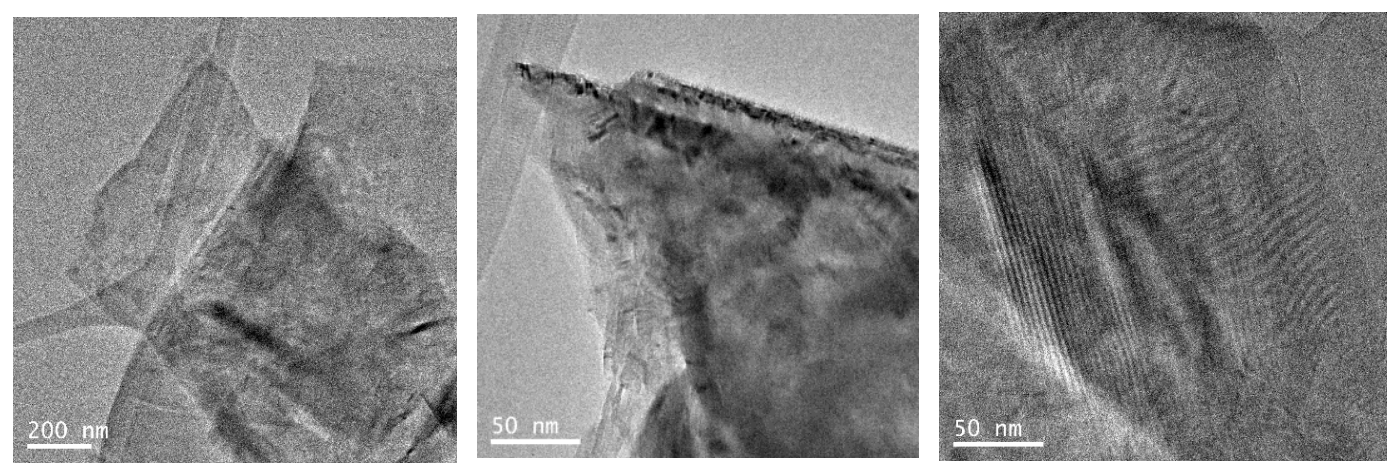

Figure 6. Characterization Method 3 using CAL-B. A) SEM Images. B) TEM images.

By analysis of the biographene samples with Scanning electron microscopy (SEM), the method using CALB seems to generate a material with larger size if we compared with the method using TLL (Figure 7). The TEM images of biographene-1 using CALB showed the presence of defects in the surface of the graphene sheets and also multiple layers superposition is seen in a disordered manner (Figure 6B). However, TEM images of biographene-1 using TLL showed a surface with lower defects and the layers present in the graphene were approximately $2-3$, which is perfectly appreciated in Figure 7B (50 $\mathrm{nm})$.

Considering these excellent results, a fourth method adding the enzyme directly from the beginning (sonication plus lipase immobilization in one-pot) was attempted (Figure 8). 
A
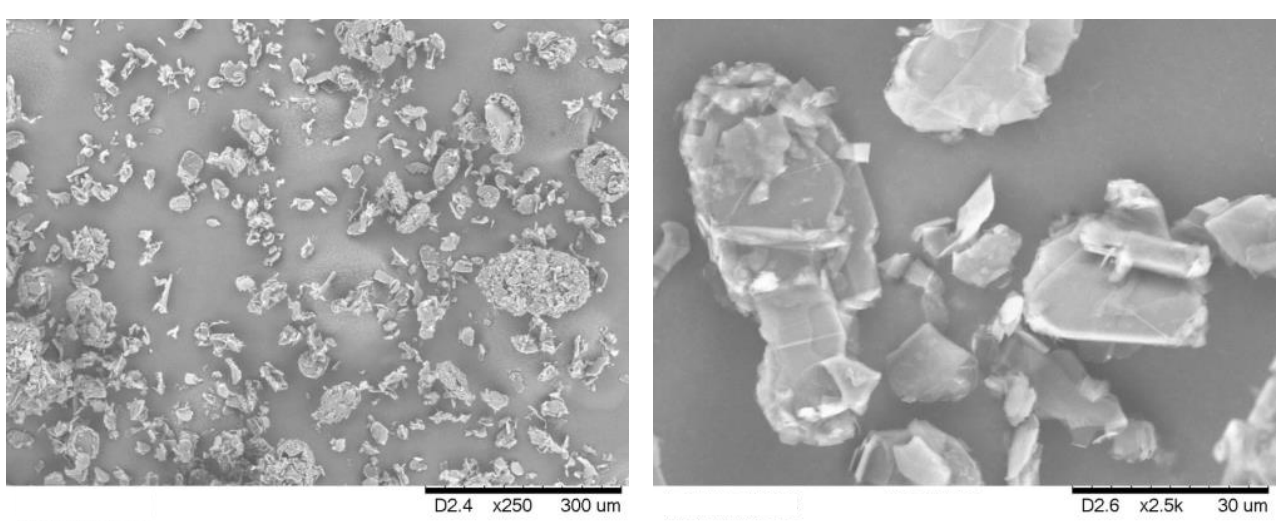

B
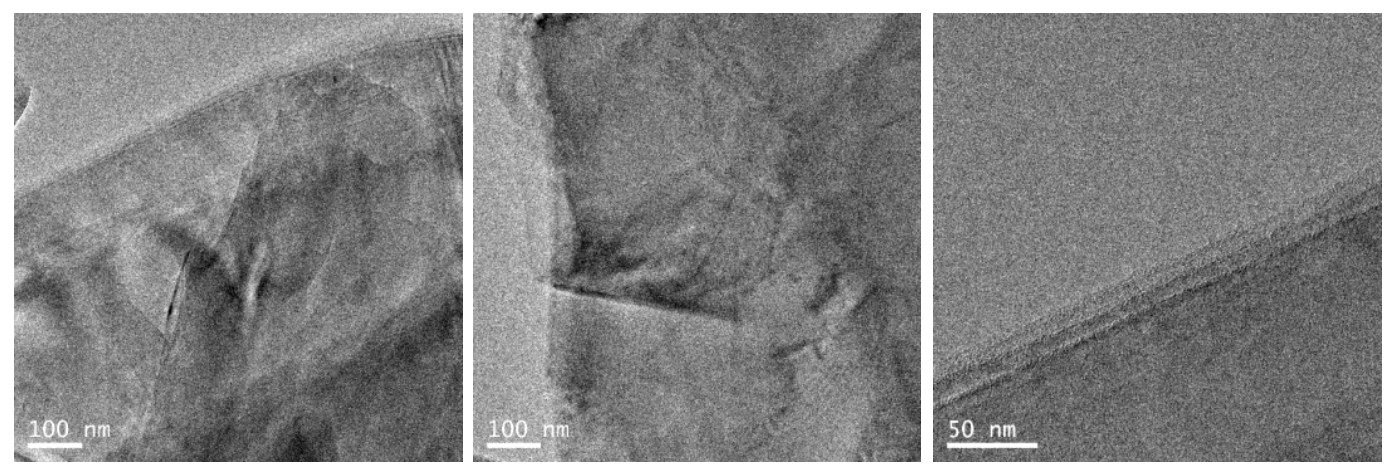

Figure 7. Characterization Method 3 using TLL. A) SEM Images. B) TEM images.

The lipase must be introduced between the sheets of graphene while the ultrasound weakens this interaction between sheets separating them completely. The strategy was applied using shorter and longer sonication times. In this case also both lipases were tested.
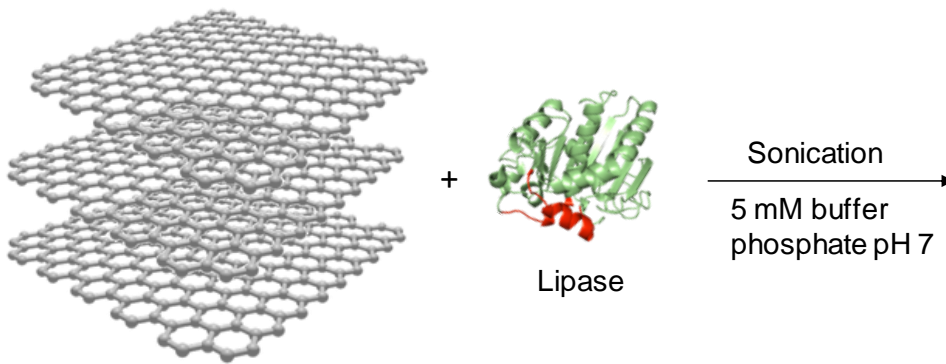

Graphite

Figure 8. Schematic representation of Biographene-2 preparation by method 4 . 
The best results were obtained using the longest strategy for mechanical sonication described in method 2 but in the presence of the enzyme. However, in both cases yields of black solid around $50 \%$ were obtained.

TEM analysis demonstrated differences between using one lipase or the other (Figure 9). In the case of CAL-B (Figure 9A.1) the particle size was slightly larger than in TLL (Figure 9A.2). In addition, in the SEM images (Figures 9B.1 and 9B.2) different morphologies were observed between them.

However, when comparing this method with the aforementioned method 3 , the sizes obtained were much larger than in method 3, together with a higher density of sheets.

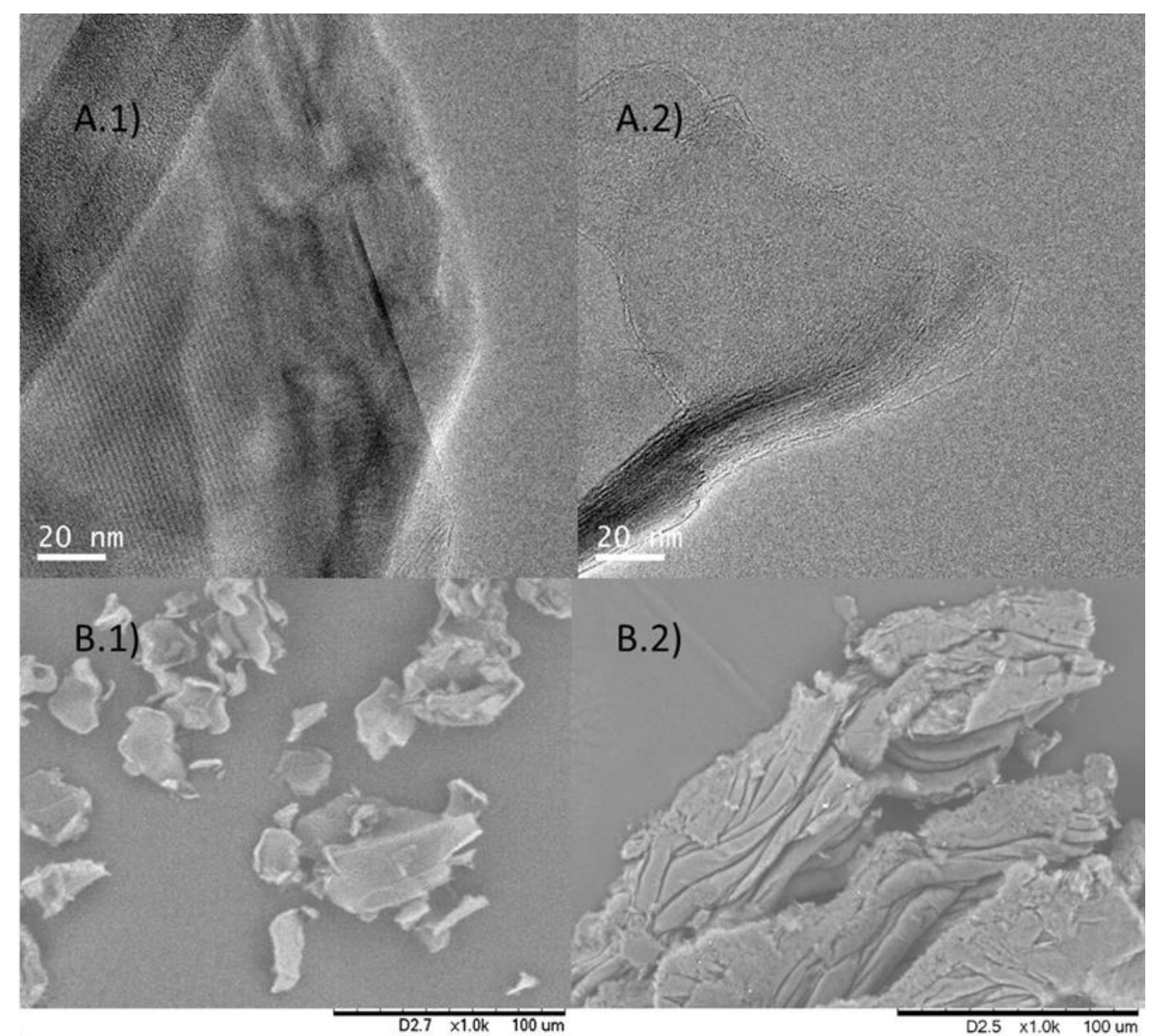

Figure 9. Characterization Method 4. A) TEM images: A.1) CAL-B, A.2) TLL; B) SEM images: B.1) CAL-B, B.2) TLL. 
In order to determine and confirm the formation of graphene based materials in all the cases and to know the graphene quality and the number of layers, Raman experiments were performed (Figure 10). The results confirmed that in all cases graphene is obtained but clear differences between methods and between enzymes were found.

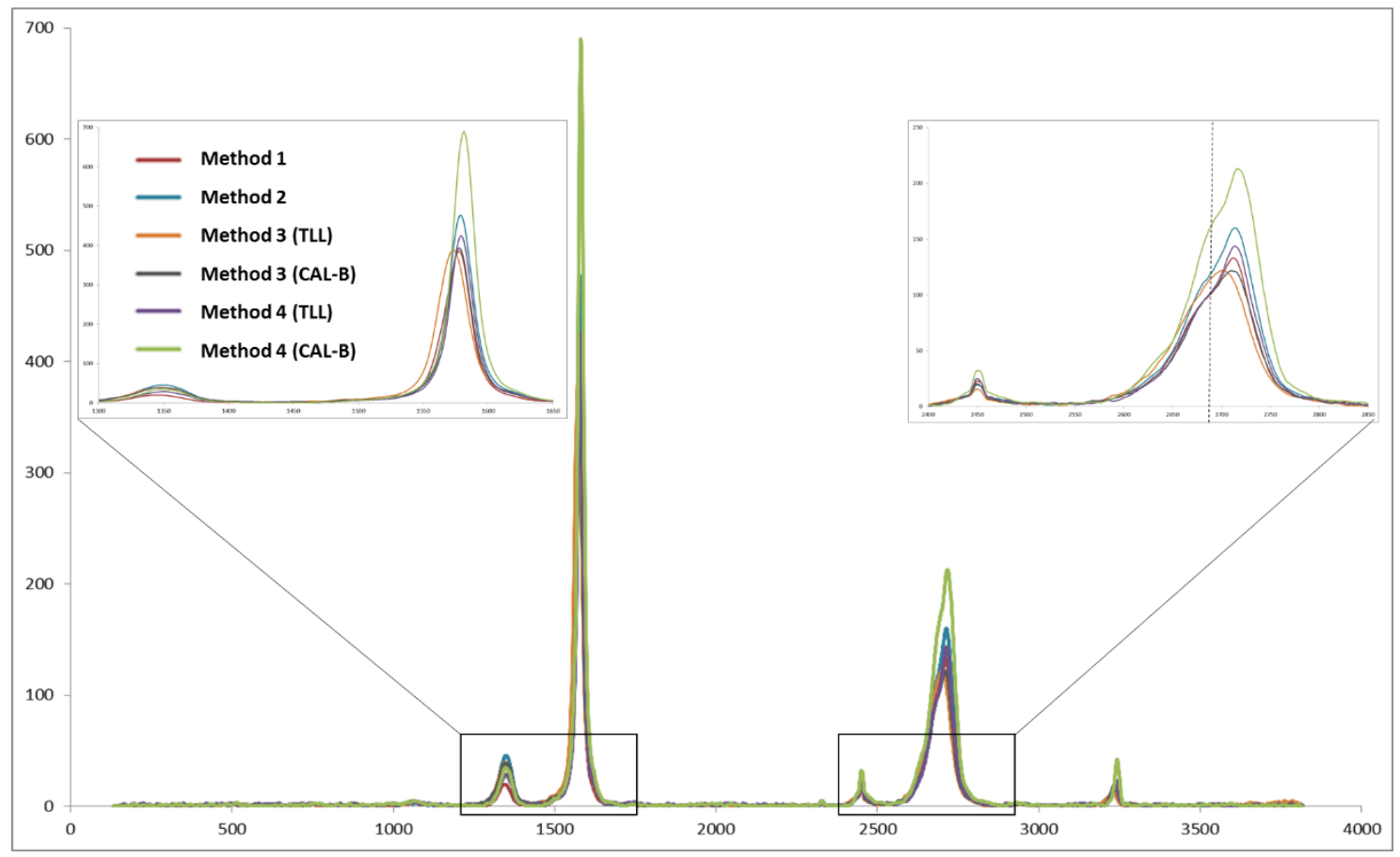

Figure 10. Raman spectra of the different methods of obtaining bio-graphene.

The TLL in method 3 showed the best results according to the Raman band range of 2600$2750 \mathrm{~cm}-1$, which clearly showed that in this case a few-layer graphene (with a number of layer between 2 and 6) is obtained, because peak at $2710 \mathrm{~cm}^{-1}$ is mainly present. ${ }^{39} \mathrm{In}$ the other cases mixtures of graphene with different number of layers from 2 to 6 are present. These results reinforce the idea that better results are found using lipase with higher hydrophobicity as TLL in comparison with CAL-B. 


\section{Conclusions}

We have developed for the first time a very simple, rapid, low-cost and environmentally safe process to produce undamaged graphene sheets from very cheap graphite flakes in water and room temperature.

The novelty consists in the application of a biomolecule, a lipase, an enzyme with a very high selectivity to interact with hydrophobic surfaces, fixing the open conformation which permits the location of lipases in open conformation uniformly distributed on the graphene sheets surface, avoiding the interactions between sheets, generating an exquisite bioexfoliation of graphite. Two different enzymes with different methods were employed and the use of Themomyces lanuginosus lipase (with a very high hydrophobic area) gave the best results. It was possible to produce bilayer graphene at $80 \%$ yield, obtaining 800 $\mathrm{mg}$ of graphene per gram of graphite flakes by a very simple way. Also this produced better quality (i.e. less damaged) biographene than commercial GO or rGO tested.

This is very important from industrial point of view, because economically the advantage is clear, $1 \mathrm{~g}$ of graphite flakes costs 3.36 cents of euro (product from SIGMA, $2.5 \mathrm{~kg}$ to 84 euros), whereas $1 \mathrm{~g}$ of graphene oxide costs 524 euros (SIGMA), that means 15000 times cheaper. Another advantage of this produced biographene is that it is already functionalized, because the enzyme presents a high amount of carboxylic groups in the area exposed to the solvent. Therefore, this strategy could be a high potential in the application of graphene in different areas. 


\section{Conflicts of interest}

The authors declare no conflict of interests.

\section{Acknowledgments}

This research was supported by GRO PROGRAM 2017, SAMSUNG L.S and MCIUN and FEDER (Project RTI2018-095291-B-I00). The authors also thank the Spanish National Research Council (CSIC). We thank Ramiro Martínez from Novozymes. 


\section{References}

1) A.K. Geim, Science, 2009, 324, 1530-1534.

2) Q.H. Wang, K. Kalantar-Zadeh, A. Kis, J.N. Coleman and M.S. Strano, Nat. Nanotechnol., 2012, 7 , 699-712.

3) S. Bolisetty, M, Peydayesh and R. Mezzenga, Chem. Soc. Rev., 2019, 48, 463487.

4) K.S. Novoselov, V.I. Fal'Ko, L. Colombo, P.R. Gellert, M.G. Schwab and K.A Kim, Nature, 2012,490,192-200.

5) F. Schwierz, Nat.Nanotechnol., 2010,5, 487-496.

6) A.A. Balandin, Nat. Mat., 2011,10,569-581.

7) J.B. Goodenough and K.-S. Park, J. Am. Chem. Soc., 2013,135,1167-1176

8) F. Bonaccorso, L. Colombo, G. Yu, M. Stoller, V. Tozzini, A.C. Ferrari, R.S. Ruoff and V. Pellegrini, Science, 2015,347, art. no. 1246501

9) K. Yang, L. Feng, X. Shi and Z. Liu, Chem. Soc. Rev., 2013,42, 530-547.

10) K.F. Mak and J. Shan, Nat. Photonics, 2016,10, 216-226.

11) S. Yao and Y. Zhu, Adv. Mat., 2015,27,1480-1511.

12) S. Manzeli, D. Ovchinnikov, D. Pasquier, O.V. Yazyev and A. Kis, Nat. Rev.Mat., 2017,2, art. no. 17033,

13) D. Chen, H. Zhang, Y. Liu and J. Li, Graphene and its derivatives for the development of solar cells, photoelectrochemical, and photocatalytic applications. Energy Environ. Sci., 2013,6,1362-1387.

14) K. S. Novoselov, E. McCann, S. V. Morozov, V. I. Falko, M. I. Katsnelson, U. Zeitler, D. Jiang, F. Schedin and A. K. Geim, Nat. Phys., 2006, 2, 177-180.

15) X. Zhou and Z. Liu, Chem. Commun., 2010, 46, 2611. 
16) V. Georgakilas, M. Otyepka, A.B. Bourlinos, V. Chandra, N. Kim, K.C. Kemp, P. Hobza, R. Zboril and K.S. Kim, Chem. Rev., 2012,112, 6156-6214.

17) S. Park and R. S. Ruoff, Nat. Nanotechnol., 2009, 4, 217.

18) V. C. Tung, M. J. Allen, Y. Yang and R. B. Kaner, Nat.Nanotechnol, 2009, 4, 25.

19) A. M. Gravagnuolo, E. Morales-Narváez, S. Longobardi, E. T. da Silva, P. Giardina and A. Merkoçi, Adv. Funct. Mater, 2015, 25, 2771.

20) H. J. Shin, K. K. Kim, A. Benayad, S. M. Yoon, H. K. Park, I. S. Jung, M.H. Jim, H.K. Jeong, J.M. Kim and J.Y. Choi, Adv. Funct. Mater., 2009, 19, 1987.

21) P. Wei, T. Gan and K.Wu, Sensors and actuators. , 2018, 274, 551-559.

22) V. León, A. M. Rodriguez, P. Prieto, M. Prato and E. Vázquez, ACS Nano 2014, 8, 563-571.

23) S. Zhao, S. Xie, Z. Zhao, J. Zhang, L. Li and Z. Xin, ACS Sust. Chem. Eng.2018, 6, $7652-7661$.

24) H. Zhuo, X. Zhang, L. Wang, Q. Lu and D.L. Kaplan, ACS Sust. Chem. Eng. 2018, 6, $12261-12267$.

25) Y.-Z. Wang, T. Chen, H.-H. Liu, X.-C. Wang and X.-X. Zhang, J. Nanosci. Nanotechnol., 2019, 9, 2078-2086

26) B.K. Salunke and B. S. Kim, RSC Adv., 2016,6, 17158

27) A. Pattammattel and C. V. Kumar, Adv. Funct. Mater. 2015, 25, 7088-7098.

28) P. Laaksonen, M. Kainlauri, T. Laaksonen, A. Shchepetov, H. Jiang, J. Ahopelto and M.B. Linder, Angew. Chem. Int. Ed, 2010, 49, 4946-4949.

29) M. B Linder, G. Szilvay, T. Nakari-Setälä and M. Penttilä. FEMS Microbiol. Rev. 2005, 29,877-896.

30) H. Van Tilbeurgh, M.P. Egloff, C. Martinez, N. Rugani, R. Verger and C. Cambillau, Nature. 1993, 362, 814. 
31) M. Marciello, M. Filice, J. M. Palomo, Catal Sci.Technol. 2012, 2, 1531-1543.

32) G. Fernández-Lorente, Z. Cabrera, C. Godoy, R. Fernandez-Lafuente, J. M. Palomo, J.M. Guisan, Process Biochem. 2008, 43, 1061-1067.

33) J.M. Palomo, G. Munoz, G. Fernandez-Lorente, C. Mateo, R. Fernandez-Lafuente and J. M. Guisan, J. Mol. Catal. B: Enzym., 2002, 19-20, 279.

34) J. M. Palomo, M. Fuentes, G. Fernández-Lorente, C. Mateo, J.M. Guisán and R. Fernández-Lafuente, Biomacromolecules.2003,4,1-6.

35) G. Fernández-Lorente, J. M. Palomo, Z. Cabrera, J. M. Guisan and R. FernandezLafuente, Enzyme Microb. Technol, 2007, 41,565-569.

36) S. Hermanová, M. Zarevúcká, D. Bouša, M. Mikulics and Z. Sofer, App. Mat. Today, 2016, 5, 200-208

37) M. Mathesh, B. Luan, T.O. Akanbi, J.K. Weber, J. Liu, C.J. Barrow, R. Zhou and W. Yang, ACS Catal., 2016, 6, 4760-4768.

38) S. Reich and C. Thomsen, Phys. Eng. Sci. 2004, A362, 2271-2288

39) D. Graf, F. Molitor, K. Ensslin, C. Stampfer, A. Jungen, C. Hierold and L. Wirtz. Nano Lett. 2007, 7,238-242. 2a 